\title{
Evaluation of Carbon Nanotube Fiber Microelectrodes for Neurotransmitter Detection: Correlation of Electrochemical Performance and Surface Properties
}

\author{
Cheng Yang ${ }^{1 \ddagger}$, Elefterios Trikantzopoulos ${ }^{1 \ddagger}$, Christopher B. Jacobs ${ }^{2}$, B. Jill Venton ${ }^{1, *}$ \\ ${ }^{1}$ Department of Chemistry, University of Virginia, Charlottesville, Virginia 22904, United States \\ ${ }^{2}$ Center for Nanophase Materials Sciences, Oak Ridge National Laboratory, 1 Bethel Valley \\ Road, Oak Ridge, Tennessee 37831, United States \\ ${ }^{*}$ Corresponding author: email: jventon@virginia.edu, Phone 434-243-2132 \\ ${ }^{\ddagger}$ These authors contributed equally to this work.
}




\section{Abstract}

Fibers made of CNTs are attractive microelectrode sensors because they can be directly fabricated into microelectrodes. Different protocols for making CNT fibers have been developed, but differences in surface structure and therefore electrochemical properties that result have not been studied. In this study, we correlated the surface and electrochemical properties for neurochemical detection at 3 types of materials: CNT fibers produced by wet spinning with (1) polyethylenimine (PEI/CNT) or (2) chlorosulfonic acid (CA/CNT), and (3) CNT yarns made by solid-based CNT drawing. CNT yarns had well-aligned, high purity CNTs, abundant oxygen functional groups, and moderate surface roughness which led to the highest dopamine current density $\left(290 \pm 65 \mathrm{pA} / \mathrm{cm}^{2}\right)$ and fastest electron transfer kinetics. The crevices of the CNT yarn and PEI/CNT fiber microelectrodes allow dopamine to get momentarily trapped during fast-scan cyclic voltammetry detection, leading to thin-layer cell conditions and a response that was independent of applied waveform frequency. The larger crevices on the PEI/CNT fibers led to a slower time response, showing too much roughness is detrimental to fast detection. CA/CNT fibers have a smoother surface and lower currents, but their negative surface charge results in high selectivity for dopamine over uric acid or ascorbic acid. Overall, small crevices, high conductivity, and abundant oxygen groups led to high sensitivity for amine neurotransmitters, such as dopamine and serotonin. Thus, different surfaces of CNT fibers result in altered electrochemical properties and could be used in the future to predict and control electrochemical performance.

Key Words: CNT Fiber, Microelectrode, Surface Properties, Neurotransmitter, Fast-Scan Cyclic Voltammetry. 


\section{Introduction}

Carbon nanotubes (CNTs) have been used as an electrode material for more than a decade. CNTs are attractive for making microelectrodes because of their large surface area, abundant electroactive sites for biomolecules, fast electron transfer kinetics, and minimal electrode fouling.[1-4] Moreover, the small dimensions of CNTs limit tissue damage for in vivo implantation while maintaining high detection sensitivity due to the high surface-to-volume ratio.[5,6] The commonly used methods to modify CNTs on carbon fiber or metal microelectrodes include dip coating[7], self-assembled CNTs[4], and directly growing CNTs on substrates.[8,9] Although many of these methods lead to outstanding electrochemical performance, future applications are limited by the cumbersome fabrication that often requires specialized equipment and the relatively low reproducibility. Recently, development of highperformance macro-structures of CNTs, such as CNTs fibers, have led to research on their applications in bio-sensing, since fibers can be handled much more conveniently than individual CNTs.[10,11] In particular, CNT fibers can be directly fabricated into electrodes, in a manner similar to carbon fiber microelectrodes (CFMEs), dramatically simplifying the fabrication process and improving the reproducibility.[5]

Two distinct protocols have been developed for manufacturing CNT fibers: wet-spinning a suspension of CNTs into a bath or direct spinning a fiber from a CNT forest. In solution based wet spinning, CNTs are either dissolved or dispersed in a fluid, extruded out of a spinneret, and coagulated into a solid fiber by extracting the dispersant.[12,13] Neat CNT fibers can be spun into a polymer bath, such as poly(vinyl alcohol) (PVA)[14] or poly(ethylene)imine (PEI)[15], in which the polymer molecules act as coagulators for CNTs to enhance the intertube interactions. Another variation of the original wet spinning method uses acid as a solvent, simplifying the process by avoiding surfactants in the CNTs dispersion.[14,16] CNT fibers fabricated by wet spinning with chlorosulfonic acid, which is the only known CNT solvent[17,18], have the highest 
conductivity among CNTs fibers.[13] The alternative fiber production route employs a solid state process where CNTs are either directly spun as a fiber from the synthesis reaction zone[19] or from a CNT forest grown on a solid substrate[20]. CNT yarns, generated by drawing one end of the vertically aligned CNT arrays, are a favorable electrode material because of their wellaligned CNTs and improved conductivity.[21,22] While several studies have tested the electrochemical properties CNT yarn or PEI/CNT fiber microelectrodes[5,23,24], there are no reports of CA/CNT fibers for biological applications. In addition, no studies have compared different CNT fibers or correlated their surface and electrochemical properties.

This work is the first to compare different CNT fiber based microelectrodes (PEI/CNT fiber, CA/CNT fiber, and CNTs yarn microelectrodes). CNT yarn microelectrodes provide the highest sensitivity to dopamine and fastest electron transfer kinetics because of the well-aligned, high purity CNTs, abundant oxygen containing functional groups at the surface, and small crevices to trap dopamine for short times. CA/CNT fibers, as a novel electrode material, provide high selectivity to dopamine over uric acid and ascorbic acid because of their negatively charged surface. In addition, the dopamine signal did not change significantly with waveform repetition frequency at CNT yarn and PEI/CNT fiber microelectrodes using fast scan cyclic voltammetry (FSCV), due to thin-layer cell conditions where the dopamine gets trapped in the crevices. However, the larger crevices on the PEI/CNT fibers lead to a slower time response, showing too much roughness can be detrimental to fast temporal response. This study facilitates a better understanding of how surface properties lead to electrochemical properties, which will enable future predictions of electrochemical properties based on the surface characteristics of carbon nanomaterial-based microelectrodes. 


\section{Experimental Section}

\section{PEI/CNT Fiber and CA/CNT Fiber Preparation}

PEI/CNT fibers were formed as previously described.[15] HiPCo ${ }^{\circledR}$ SWCNTs (1.2 w\%) were suspended in water with sodium dodecylbenzenesulfonate (1.2 w\%) and were pumped at a rate of $0.5 \mathrm{~mL} / \mathrm{min}$ through a $30 \mathrm{G}$ syringe needle into a rotating solution of $40 \% \mathrm{PEI}$ (branched, MW = 50 000-100 000, MP Biomedicals, LLC, Santa Ana, CA) in methanol. The rotating stage was a custom modified ceramics wheel with variable speed (set to $25 \mathrm{rpm}$ here, Speedball, Statesville, NC). The CNT ribbons were subsequently purified in methanol. The diameter of $\mathrm{CA} / \mathrm{CNT}$ and PEI/CNT fibers is dependent on the flow rate of the syringe pump and the rotation speed $(25 \mathrm{rpm})$ of the stage (Speedball, Statesville, NC). CNT fibers were dried in air and then $180^{\circ} \mathrm{C}$ for $1 \mathrm{~h}$.

CA/CNT fibers were formed as previously described.[13] HiPCo CNTs were dissolved in chlorosulfonic acid at a concentration of $1.2 \mathrm{w} \%$ (same as for PEI/CNT) to form a spinnable liquid crystal dope (solution of aligned CNTs and chlorosulfonic acid)[12,13]. With CNT concentration more than $1.2 \mathrm{w} \%$, the $\mathrm{CA} / \mathrm{CNT}$ fiber microelectrodes produced were electrochemically noisy and unstable). The dope was extruded through a spinneret at a rate of $0.5 \mathrm{~mL} / \mathrm{min}$ into acetone to remove the acid. The fibers were dried in room conditions for five hours under constant stretching and tensioning from both ends.

\section{CNT Fiber and Yarn Microelectrode Fabrication}

A $0.68 \mathrm{~mm} \mathrm{ID} \mathrm{\times 1.2} \mathrm{mm} \mathrm{OD} \mathrm{(A-M} \mathrm{Systems,} \mathrm{Carlsborg,} \mathrm{WA)} \mathrm{glass} \mathrm{capillary} \mathrm{was} \mathrm{pulled}$ with a glass pipet puller and cut to have an opening diameter of $\sim 50 \mu \mathrm{m}$. A piece of either PEI/CNT fiber, CA/CNT fiber, or CNT yarn (15-25 $\mu \mathrm{m}$ in diameter, 1-2 cm long, General Nano, LLC, Cincinnati, OH) was inserted into the glass pipette. The electrodes were epoxied with Epon Resin 828 (Miller-Stephenson, Danbury, CT) mixed with 14 weight\% 1,3phenylenediamine hardener (Sigma-Aldrich, St. Louis, MO) heated to $85^{\circ} \mathrm{C}$. The epoxied 
electrodes were cured overnight at room temperature and then heated at $100^{\circ} \mathrm{C}$ for $2 \mathrm{~h}$ and at $150^{\circ} \mathrm{C}$ overnight. Microelectrodes were polished at a $90^{\circ}$ angle on a fine diamond abrasive plate (Sutter Instruments model BV-10, Novato, CA). Disk CFMEs were fabricated using 7- $\mu$ mdiameter T-650 carbon fibers (Cytec Technologies, Woodland Park, NJ).[23]

\section{Results and Discussion}

\section{Surface Comparison of CNT Fiber Microelectrodes}

CNT fibers were fabricated as microelectrodes and polished vertically at the tips, which allows physical characterizations of the electroactive surface, instead of the entire bulk fiber. CA/CNT and PEI/CNT fibers were constructed by wet spinning procedures, as reported previously.[13,15] The diameter of CA/CNT and PEI/CNT fibers is dependent on the flow rate of the syringe pump and the rotation speed $(25 \mathrm{rpm})$ of the stage. The diameters of both fibers were $\sim 20 \mu \mathrm{m}$, as shown in Figure 1A and B, which is similar to the diameter of CNT yarn (Fig. 1C). Chlorosulfonic acid is the first true solvent for CNTs.[17,25] A solution, rather than a suspension, of CNTs could lead to improved CNTs alignment and packing of the CA/CNT fibers.[13] The CA/CNT fiber retained its circular shape and coagulated in a uniform manner (Fig. 1A), with a substructure of well packed CNT super-ropes, approximately $200-500 \mathrm{~nm}$ in diameter, connected to one another (Fig. 1D). PEI/CNT fibers are ribbon-like gel fibers that partially collapse when drying to form tubular structures.[15] The surface of the PEI/CNT fiber is primarily composed of SWCNTs with distinct regions of PEI that are not fully removed during rinsing. The outer fiber surface is corrugated, with the axis of corrugation lying approximately parallel to the fiber axis (Fig. 1B). These corrugations with diameters about $0.5-1 \mu \mathrm{m}$ are typically observed along the outer surface of fibers and could be also seen on the polished microelectrode surface (Fig. 1E). The CNT yarn (Fig. 1C) was approximately $20 \mu \mathrm{m}$ in diameter and was spun from a forest of $40-50 \mathrm{~nm}$ diameter individual multiwalled CNTs.[24] The CNTs bundles, which are the ends of CNTs, show the relatively rough surface on CNT yarn 
microelectrode tip (Fig. 1F). The surfaces of the PEI/CNT fiber and CNT yarn are much rougher than the surface of CA/CNT fiber.
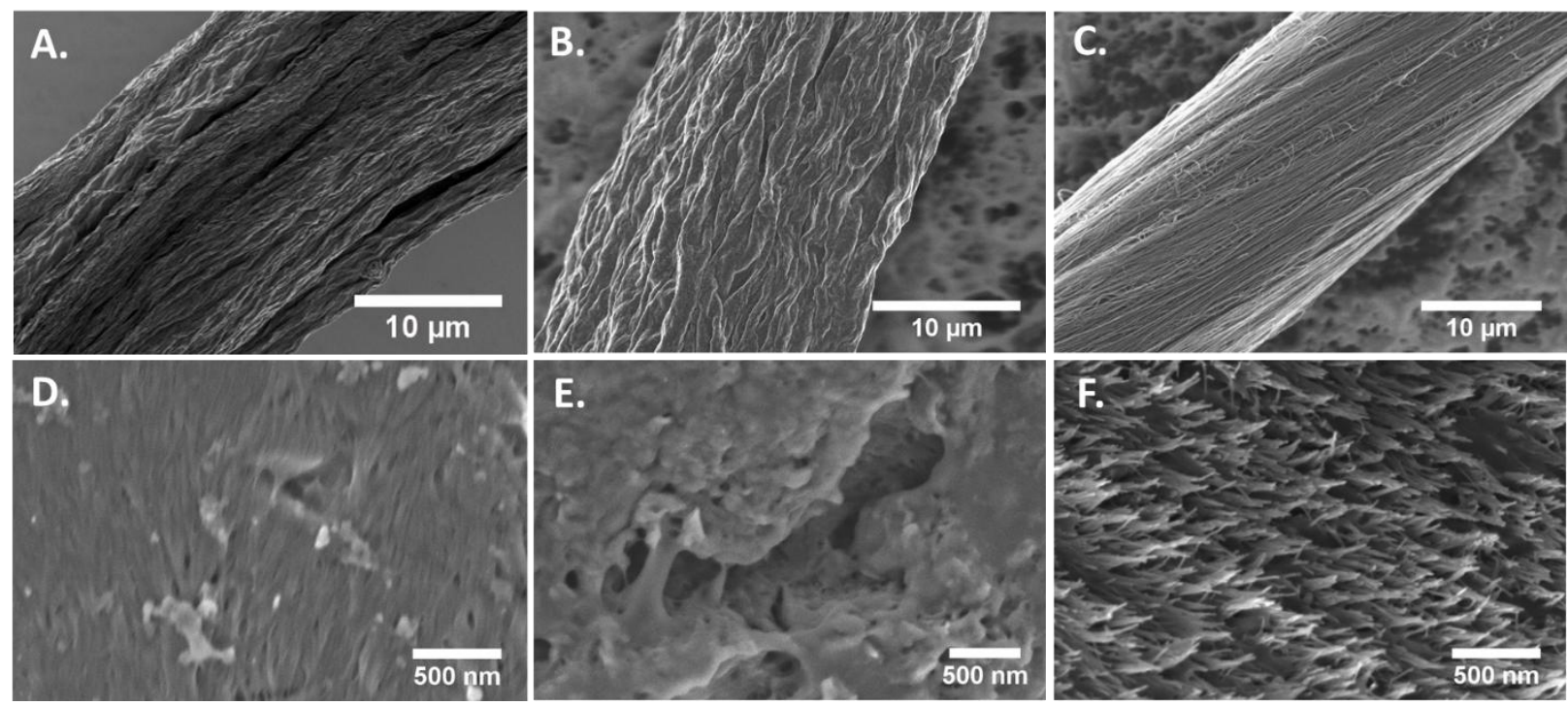

Figure 1. Surfaces of microelectrodes. SEM images of the (A) CA/CNT fiber, (B) PEI/CNT fiber, and (C) CNT yarn sidewall, with the diameters about $20 \mu \mathrm{m}$. Scale bar: $10 \mu \mathrm{m}$. Top-view SEM images at the tips of polished (D) CA/CNT fiber, (E) PEI/CNT fiber, and (F) CNT yarn microelectrodes. Scale bar: $500 \mathrm{~nm}$.

Table 1. Elemental Contents at the tips of Microelectrodes Measured by EDS

\begin{tabular}{|c|c|c|c|c|c|c|}
\hline & $\mathrm{C} / \%$ & $\mathrm{O} / \%$ & $\mathrm{Fe} / \%$ & $\mathrm{~S} / \%$ & $\mathrm{Cl} / \%$ & $\mathrm{~N} / \%$ \\
\hline CA/CNT Fiber & $79 \pm 2$ & $11 \pm 1$ & $2.1 \pm 0.1$ & $3 \pm 1$ & $2 \pm 1$ & $\mathrm{~N} / \mathrm{A}$ \\
\hline PEI/CNT Fiber & $66.8 \pm 0.2$ & $12.6 \pm 0.5$ & $3.2 \pm 0.2$ & $0.43 \pm 0.05$ & $\mathrm{~N} / \mathrm{D}$ & $16.4 \pm 0.2$ \\
\hline CNT Yarn & $81 \pm 1$ & $19 \pm 1$ & $\mathrm{~N} / \mathrm{D}$ & $\mathrm{N} / \mathrm{D}$ & $\mathrm{N} / \mathrm{D}$ & $\mathrm{N} / \mathrm{D}$ \\
\hline
\end{tabular}

N/D: not detected

The elemental content was measured at the tip of polished microelectrodes by energydispersive X-ray spectroscopy (EDS), as shown in Table 1. The CA/CNT fiber has small amounts of sulfur and chlorine, which likely come from residual chlorosulfonic acid. The large amounts of nitrogen on the PEI/CNT fiber comes from PEI which was not fully removed during rinsing and the small amounts of sulfur from the sulfur-containing anionic surfactants in the 
suspension solution. PEI/CNT fibers have more residue than CA/CNT fibers. Both the CA/CNT and PEI/CNT fibers have iron impurities from HiPco SWCNTs. In contrast, the CNT yarns are relatively pure, with no polymer, surfactant residue, or iron impurities. Moreover, the oxygen content at the CNT yarn surface is significantly larger than at CA/CNT and PEI/CNT fibers (unpaired t-test, $\mathrm{p} \leq 0.01$ for both comparisons).

The conductivity was measured at each fiber using four-point probe method. The CNT yarn has highest conductivity, $1025 \pm 25 \mathrm{~S} \cdot \mathrm{cm}^{-1}(\mathrm{n}=3)$, which is in agreement with the literature value range $\left(500-1250 \mathrm{~S} \cdot \mathrm{cm}^{-1}\right) \cdot[26]$ The conductivity of our CA/CNT fibers, $618 \pm 23 \mathrm{~S} \cdot \mathrm{cm}^{-1}$, was not as good as CA/CNT fiber reported previously $\left(2900 \pm 300 \mathrm{~S} \cdot \mathrm{cm}^{-1}\right) \cdot[13]$ The lower conductivity might be caused by different SWCNTs having more iron impurity, as indicated by EDS measurements. PEI/CNT fibers have the lowest conductivity, $16.4 \pm 0.4 \mathrm{~S} \cdot \mathrm{cm}^{-1}(\mathrm{n}=3)$, similar to previous work $\left(10-30 \mathrm{~S} \cdot \mathrm{cm}^{-1}\right) \cdot[15]$ Although PEI is an ionically conductive polymer, its conductivity is not as high as pure CNTs so the residual PEI would decrease the overall conductivity. The high-to-low trend of conductivity: CNT yarn, CA/CNT fiber, and PEI/CNT fiber, is consistent with the high-to-low trends of alignment and purity of CNTs in the fibers.

\section{Electrochemical Characterization of Dopamine Detection at CNT Fiber Microelectrodes}

To investigate the electrochemical performance, the response to $1 \mu \mathrm{M}$ dopamine was tested using FSCV with a triangle waveform from -0.4 to $1.3 \mathrm{~V}$ and back at $400 \mathrm{~V} / \mathrm{s}$ and a scan repetition frequency of $10 \mathrm{~Hz}$. Figures $2 \mathrm{~A}-\mathrm{C}$ show the background subtracted cyclic voltammograms (CVs) of $1 \mu \mathrm{M}$ dopamine at disk CA/CNT, PEI/CNT fiber, and CNT yarn microelectrodes with a similar diameter $(\sim 20 \mu \mathrm{m})$. The CNT yarn microelectrode has the largest oxidation and reduction currents as well as the smallest difference in oxidation and reduction 
peak potential $\left(\triangle E_{p}\right)$ (Fig. $\left.2 \mathrm{C}\right)$. The current for the CA/CNT and PEI/CNT fiber microelectrodes is similar, but the $\Delta \mathrm{E}_{\mathrm{p}}$ is smaller at CA/CNT fibers.

To compare the electrochemical performance of microelectrodes, dopamine currents were corrected for surface area (based on their capacitive charging currents). As shown in Table 2, the current density at CNT yarns is $290 \pm 65 \mathrm{pA} / \mu \mathrm{m}^{2}$, which is significantly larger than the current density at PEI/CNT fibers (unpaired t-test, $p \leq 0.05$ ), but not statistically different than CA/CNT fibers (unpaired t-test, $p=0.2748$ ). The current density at CFMEs is $135 \pm 24$ $\mathrm{pA} / \mu \mathrm{m}^{2},[9]$ smaller than the CA/CNT fiber and CNT yarn.

Table 2. Comparison of Electrochemical Dopamine Detection

\begin{tabular}{|c|c|c|}
\hline & $\begin{array}{c}\text { Current Density } \\
\left(\mathrm{pA} / \mu \mathrm{m}^{2}\right)\end{array}$ & $\Delta \mathrm{E}_{\mathrm{p}} / \mathrm{V}$ \\
\hline CA/CNT Fiber & $210 \pm 31$ & $0.89 \pm 0.02$ \\
\hline PEI/CNT Fiber & $122 \pm 23$ & $1.04 \pm 0.02$ \\
\hline CNT Yarn & $290 \pm 65$ & $0.68 \pm 0.02$ \\
\hline
\end{tabular}

All $n=5$, Errors are standard error of mean

The $\Delta \mathrm{E}_{\mathrm{p}}$ values are significantly lower at CNT yarn than CA/CNT fiber or PEI/CNT fiber microelectrodes (Table 2, unpaired t-test, $\mathrm{p} \leq 0.0001$ for both comparisons). The $\Delta \mathrm{E}_{\mathrm{p}}$ value at CA/CNT fiber microelectrodes is significantly smaller than PEI/CNT fibers (unpaired t-test, $p \leq$ 0.01). The smaller $\Delta \mathrm{E}_{\mathrm{p}}$ might be caused by differing double-layer capacitances, uncompensated resistance, or ohmic drop.[5] However, because both the electrolyte and the size of the electrodes are similar, ohmic drop is an unlikely cause. Therefore, the smaller $\Delta \mathrm{E}_{\mathrm{p}}$ value indicates faster electron transfer kinetics, with the fastest electron transfer rates observed in order of: CNT yarn > CA/CNT fiber > PEI/CNT fiber. 
Figure 2D-F shows the current versus time curves in the response to a bolus of dopamine. CA/CNT fiber microelectrodes have a current versus time curve (Fig. 2D) similar to conventionally used CFMEs, with its time response performance falling between CNT yarn and PEI/CNT fiber microelectrodes. The time response at PEI/CNT fiber microelectrodes is slow (Fig. 2E), the signal does not plateau, and does not go back to baseline. The CNT yarn microelectrode has a relatively faster temporal response, with an average $10-90 \%$ signal rise time of $1.0 \pm 0.1 \mathrm{~s}(\mathrm{n}=3)$, compared to $1.1 \pm 0.2 \mathrm{~s}(\mathrm{n}=3)$ at CA/CNT fibers and $3.1 \pm 0.5 \mathrm{~s}$ at PEI/CNT fiber microelectrodes (Fig. 2F).

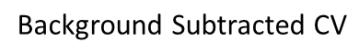

Current-time Trace
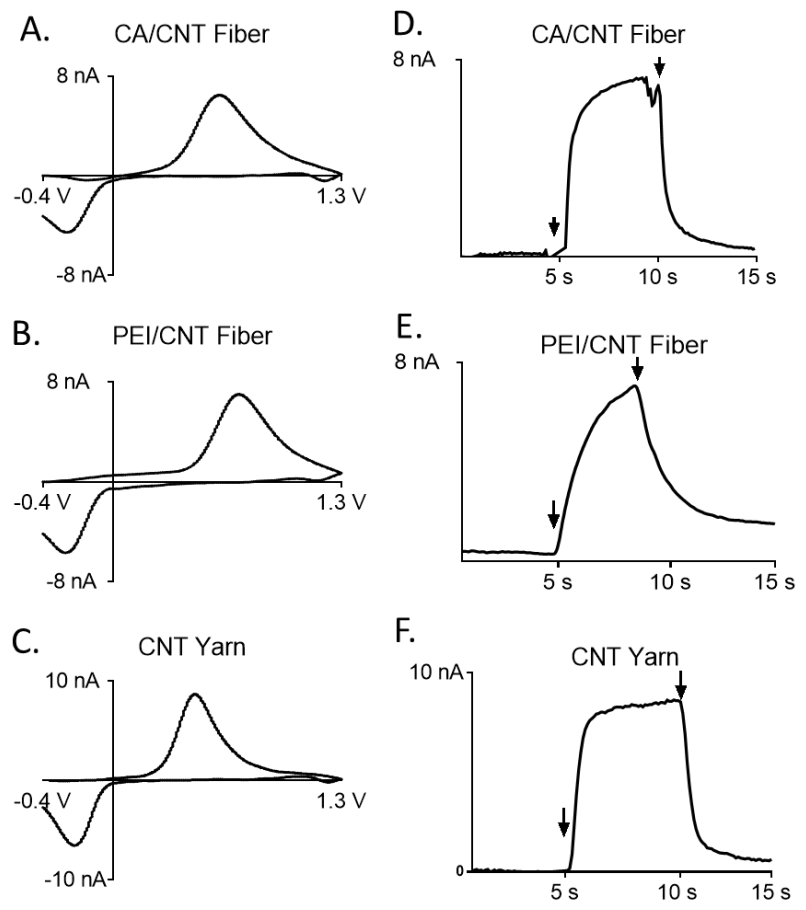

Figure 2. Electrochemical response to $1 \mu \mathrm{M}$ dopamine with a waveform of $-0.4 \mathrm{~V}$ to $1.3 \mathrm{~V}$ and back at $400 \mathrm{~V} / \mathrm{s}, 10 \mathrm{~Hz}$. (Left) Background subtracted cyclic voltammograms at (A) CA/CNT fiber, (B) PEI/CNT fiber, and (C) CNT yarn microelectrodes. (Right) Current versus time trace plot for the same electrodes measured at $0.6 \mathrm{~V}$ in flow injection experiment. The first arrow indicates the injection of dopamine bolus and the second arrow indicates the switch back to buffer.

\section{Electrochemical Characterization of Other Neurochemicals at CNT Fiber Microelectrodes}


Because many electroactive substances coexist in the brain, the selectivity of a sensor for neurotransmitters is another important factor to consider when selecting microelectrodes. We tested the electrochemical performance of different CNT fiber microelectrodes toward the detection of neurochemicals including uric acid, ascorbic acid, and serotonin. Uric acid (UA) acts as an antioxidant in cerebrospinal fluid and is an anion at physiological pH.[27] Ascorbic acid (AA), also an anion at physiological $\mathrm{pH}$, is one of the most abundant, low molecular weight antioxidants in the central nervous system.[28] UA and AA are common interferences for in vivo dopamine detection, and the extracellular levels of UA[29] and $A A[30,31]$ are several orders of magnitude higher than dopamine in the brain. Serotonin is a cationic indolamine neurotransmitter with a similar oxidation potential to dopamine.[32]

Figure $3 \mathrm{~A}$ shows $\mathrm{CVs}$ for $20 \mu \mathrm{M} \mathrm{UA}, 200 \mu \mathrm{M} \mathrm{AA}$, and $1 \mu \mathrm{M}$ serotonin at the same CA/CNT fiber, PEI/CNT fiber, or CNT yarn microelectrode. Table S1 gives the current density for different analytes at each electrode. The bar graphs (Fig. 3B) compare the ratio of oxidation currents for each neurochemical ( $20 \mu \mathrm{M} \mathrm{UA}, 200 \mu \mathrm{M} \mathrm{AA}$, or $1 \mu \mathrm{M}$ serotonin) to that of dopamine $(\mathrm{DA}, 1 \mu \mathrm{M})$. As shown in Figure 3B, the UA: DA signal ratio at CA/CNT and PEI/CNT fibers microelectrodes is significantly smaller than at CNT yarn microelectrodes (unpaired t-test, $p \leq$ 0.05 for both comparisons). Moreover, the AA: DA signal ratio is significantly smaller at CA/CNT fibers than CNT yarns (unpaired t-test, $p<0.05$ ). The CA/CNT fiber has the lowest UA and AA to DA signal ratios, indicating it has the highest selectivity for dopamine compared to UA and AA.

Serotonin is positively charged at physiological pH; however, it is also known to foul the electrode surface when applying the waveform used here.[33] At CA/CNT fibers, there is an extra peak found at $0.3-0.4 \mathrm{~V}$, which might be caused by polymerization of serotonin and indicative of fouling. The extra peak is also visible at PEI/CNT fibers, but much smaller than at CA/CNT fiber microelectrodes. In contrast, CNT yarn microelectrodes have a sharp, single oxidation peak for serotonin. The ratios of currents for serotonin to dopamine as well as the 
serotonin current densities are similar at CA/CNT and PEI/CNT fiber microelectrodes (Fig. 3B, unpaired t test, $\mathrm{p}=0.8463, \mathrm{n}=4$, Table $\mathrm{S} 1$ ). In comparison, the serotonin: DA current ratio and the serotonin current density at CNT yarn microelectrodes are significantly larger than at CA/CNT and PEI/CNT fibers (unpaired t-test, $\mathrm{p} \leq 0.0001$ for both ratio and current density comparisons). In our previous study, PEI/CNT fiber microelectrodes show promising resistance to surface fouling by serotonin,[5] with serotonin oxidation currents remaining constant over 25 consecutive serotonin injections $(1 \mu \mathrm{M}$ serotonin solution was injected for $5 \mathrm{~s}$ every $15 \mathrm{~s}$ for 25 injections). Similarly, we tested serotonin fouling at CNT yarn microelectrodes and found no decrease due to serotonin fouling (signal was $96 \%$ of initial signal after 25 injections, one-way ANOVA, $p=0.24, n=3$ ).

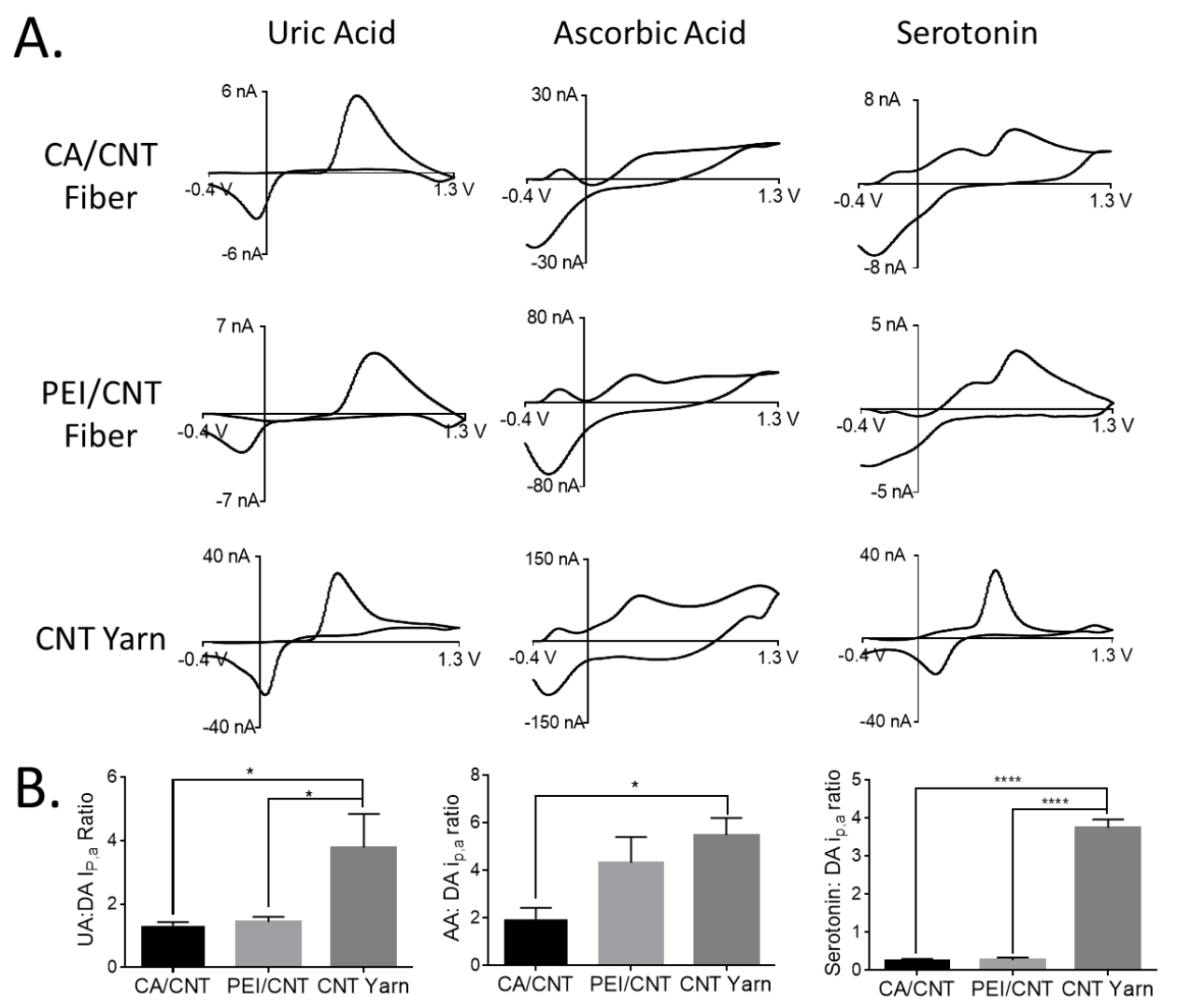

Figure 3. Detection of different neurochemicals at CNT fiber microelectrodes. (A) Detection of $20 \mu \mathrm{M}$ uric acid, $200 \mu \mathrm{M}$ ascorbic acid, and $1 \mu \mathrm{M}$ serotonin in PBS buffer at a CA/CNT fiber (top), PEI/CNT fiber (middle), or CNT yarn (bottom) microelectrode. (B) Bar graphs show the 
ratio of oxidation current for UA, AA, or serotonin compared to the oxidation current of dopamine ( $n=4$ each). ${ }^{*} p<0.05$ and ${ }^{* * *} p<0.0001$

\section{Dopamine Sensitivity at Rapid Scan Repetition Frequency}

The typical FSCV triangle waveform with a scan rate of $400 \mathrm{~V} / \mathrm{s}$ takes $8.5 \mathrm{~ms}$. The time between waveforms is $91.5 \mathrm{~ms}$ with a scan repetition frequency of $10 \mathrm{~Hz}$ but only $1.5 \mathrm{~ms}$ when the scan repetition frequency increases to $100 \mathrm{~Hz}$. The redox reaction of dopamine at the carbon based sensor surface is an adsorption-controlled process so the dopamine signal drops with decreasing time for dopamine adsorption at CFMEs.[34,35] The loss of sensitivity with rapid scan repetition frequency at carbon fibers limits the temporal resolution of the measurement. Therefore, electrode materials which have a current that is independent of the scan repetition rate would be better able to monitor rapid reactions and follow the kinetics of neurotransmission.[23]

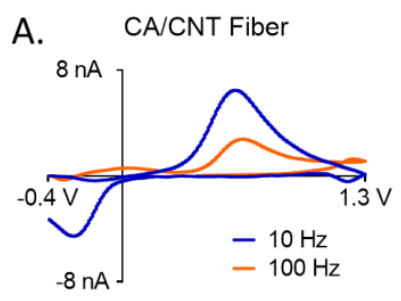

CNT Yarn

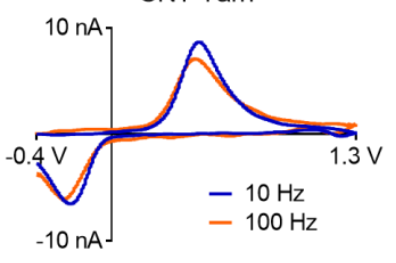

B.

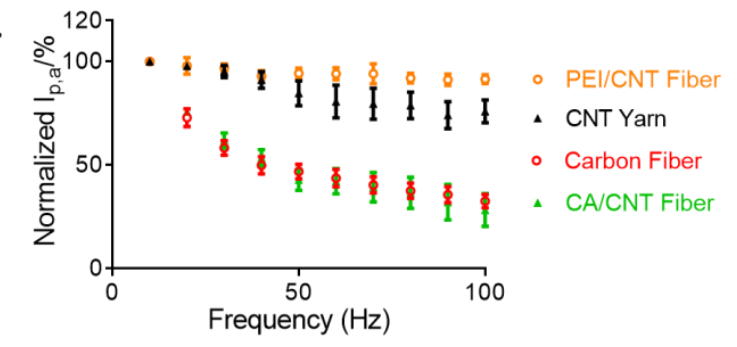

Figure 4. Effect of scan repetition frequency for $1 \mu \mathrm{M}$ dopamine detection. (A) Example CVs of $1 \mu \mathrm{M}$ dopamine at the scan repetition frequency of $10 \mathrm{~Hz}$ (blue line) and $100 \mathrm{~Hz}$ (orange line), 
with -0.4 to $1.3 \mathrm{~V}$ waveform. (B) Peak oxidation current at PEI/CNT fiber microelectrodes (blue circle, $n=4$ ), CNT yarn microelectrodes (black triangle, $n=5$ ), carbon fiber microelectrodes, (red circle, $n=5$ ), and CA/CNT fiber microelectrodes (green triangle, $n=4$ ), from top to bottom. Data is normalized to dopamine oxidation signal at different microelectrodes with scan repetition frequency of $10 \mathrm{~Hz}$.

To study the scan repetition frequency dependence, the current for $1 \mu \mathrm{M}$ dopamine was measured with scan repetition frequencies from 10 to $100 \mathrm{~Hz}$ (Figure 4A). The oxidation current is relatively stable with increasing scan frequency at PEI/CNT and CNT yarn microelectrodes, but decreases dramatically at CA/CNT fiber and CFMEs. Moreover, the oxidation/reduction peak ratio is larger at PEI/CNT fiber and CNT yarn than those at carbon fiber and CA/CNT fiber at both 10 and $100 \mathrm{~Hz}$. Figure 4B shows a significant effect of electrode type on dopamine current with different scan repetition frequencies (one-way ANOVA, $p<0.0001, n=4$ ). The oxidation current drops dramatically at CFMEs, with approximately $67 \%$ loss at $100 \mathrm{~Hz}$ compared to $10 \mathrm{~Hz}$, and the current at CA/CNT fiber microelectrodes dropped similarly, $72 \%$ at $100 \mathrm{~Hz}$ (one-way ANOVA Bonferroni post-test, $\mathrm{p}=0.9561$ ). The oxidation currents of dopamine at CNT yarn and PEI/CNT fiber microelectrodes dropped approximately $24 \%$ and $8.4 \%$ at 100 $\mathrm{Hz}$, respectively, a significantly smaller drop than CA/CNT fibers (one-way ANOVA. Bonferroni post-test, $p \leq 0.001$ and $p \leq 0.0001$, respectively) or CFMEs ( $\leq 0.001$ and $p \leq 0.0001$, respectively). The results are consistent with our previous work that the current at CNTYMEs[23], laser treated CNTYMEs[36], and PEI/CNT fiber[5] retain high sensitivity at rapid scan repetition frequency.

\section{Correlation of Electrochemical Performance and Surface Properties}

Surface properties of CNT fibers produced by different protocols have substantial influences on the electrochemical performance for the detection of neurotransmitters. The better aligned CNTs in CNT yarns and CA/CNT fibers, compared to PEI/CNT fibers, provide higher overall conductivity and better sensitivity for dopamine detection (Table 2). The extra current 
density observed at CNT yarns over CA/CNTs is attributed to the relatively higher oxygen content and less residue on CNT yarn surface (Table 1). CNT yarns have abundant oxygen containing functional groups, such as quinones, hydroxyls, and carboxylic acids, which are known to be negatively charged at physiological $\mathrm{pH}$ and have electrostatic interactions with positively charged neurotransmitters, such as dopamine.[37] The $\Delta E_{p}$, a measure of electron transfer kinetics, followed the same order as CNT alignment: CNT yarns > CA/CNT fibers > PEI/CNT fibers. In addition, the faster electron transfer rate observed at CA/CNTs compared to PEI/CNT fiber microelectrodes might be caused by less residue and higher conductivity.

The CA/CNT fiber was applied as a biomolecule microelectrode material for the first time. It provides high selectivity to dopamine over UA and AA because of the negative charge carried by the acid-doping at the fiber surface, which makes CA/CNT fibers a good microelectrode material for dopamine detection in the presence of large amount of interferences. CNT yarn microelectrodes have dramatically higher serotonin sensitivity compared to PEI/CNT and CA/CNT fiber microelectrodes. The abundant oxygen containing functional groups provide electroactive sites with negative charges, which are good for adsorption of serotonin, a cation at physiological $\mathrm{pH}$, similar to dopamine.

The waveform frequency independent property of CNT yarn and PEI/CNT fiber microelectrodes allows highly sensitive detection of dopamine at a scan repetition frequency of $100 \mathrm{~Hz}$, which is one order of magnitude faster than the conventionally applied frequency. To understand adsorption strength, we applied a Langmuir adsorption isotherm to model the adsorption and desorption of dopamine as well as the oxidation product of dopamine, dopamine-o-quinone (DOQ).[9] Plotting percent surface coverage versus concentration for the anodic peaks (Fig. S1 A-C) and cathodic peaks (Fig. S1 D-F) gives information about dopamine and DOQ adsorption/desorption, respectively. The adsorption/desorption equilibrium constants for both dopamine $\left(\beta_{\mathrm{DA}}\right)$ and $\mathrm{DOQ}\left(\beta_{\mathrm{DOQ}}\right)$ at CNT yarn microelectrodes are larger than those at 
CA/CNT fiber and PEI/CNT fiber microelectrodes (Table 3, one-way ANOVA, Brown-Forsythe test, $p<0.01$, and $p<0.0001$ for $\beta_{\mathrm{DA}}$ and $\beta_{\mathrm{DOQ}}$, respectively), indicating dopamine and $\mathrm{DOQ}$ adsorption is stronger on the CNT yarn surface than the other two CNT fibers. The strong adsorption is likely due to the abundant oxygen containing functional groups and fewer impurities (both polymer residues and iron catalyst) at CNT yarns. The ratio of $\beta_{D A}$ to $\beta_{D O Q}$ is used to compare the adsorption equilibria differences between dopamine and DOQ. A ratio larger than 1 means that dopamine adsorption is stronger than DOQ and that DOQ has a greater tendency to desorb, leading to smaller reduction/oxidation peak current ratios. In comparison, a ratio of 1 indicates dopamine and DOQ have similar adsorption strength, so more DOQ will remain adsorbed and be reduced back to dopamine, amplifying dopamine detection on the next scan and giving the material a frequency independent property. The $\beta_{D A} / \beta_{D O Q}$ ratio is about 1 at PEI/CNT fibers and CNT yarn microelectrodes, signifying the oxidation and reduction peak currents are similar (Fig. 2A-C, Fig. 4A). The ratio of equilibrium constants for CA/CNT fiber microelectrodes is greater than 1 and significantly larger than PEI/CNT fiber (unpaired ttest, $p \leq 0.01$ ) or CNT yarn microelectrodes (unpaired t-test, $p \leq 0.05$ ), but similar to CFMEs (with ratio of $1.23 \pm 0.04$ )[9]. Thus, $\mathrm{DOQ}$ is more likely to desorb from the surface of CA/CNT fibers, leading to smaller reduction peak. These data agree with previous modeling of CNT yarn and CNT-metal microelectrodes, which showed the similar $\beta_{\mathrm{DA}}$ to $\beta_{\mathrm{DOQ}}$ values lead to frequency independent currents.[9,23]

Table 3. Average Equilibrium Constants ( $\beta$ ) for Dopamine and Dopamine-o-quinone

\begin{tabular}{|c|c|c|c|}
\hline Material & $\beta_{\mathrm{DA}}\left(\times 10^{3}\right)$ & $\beta_{\mathrm{DOQ}}\left(\times 10^{3}\right)$ & $\beta_{\mathrm{DA}} / \beta_{\mathrm{DOQ}}$ \\
\hline CA/CNT Fiber & $10 \pm 1$ & $8 \pm 1$ & $1.27 \pm 0.07$ \\
\hline PEI/CNT Fiber & $14 \pm 1$ & $14 \pm 1$ & $0.97 \pm 0.04$ \\
\hline CNT Yarn & $19 \pm 3$ & $19 \pm 4$ & $1.08 \pm 0.09$ \\
\hline
\end{tabular}

All $n=4$, Errors are standard error of mean. 
Surface roughness also influences the electrochemical properties.[36] Dopamine and DOQ are more likely to get trapped in the rough surface of CNT yarn and PEI/CNT fiber (Fig. 1E and $1 \mathrm{~F}$ ), leading to a reduction peak that is of similar magnitude to the oxidation peak. Surface roughness also influences the scan frequency dependence. At smoother surfaces, such as the $\mathrm{CA} / \mathrm{CNT}$ electrode, when DOQ desorbs, it can easily diffuse away from the electrode surface. At the rough microelectrode surfaces of CNT yarns or PEI/CNT fibers, the response would be similar to that in a thin layer cell, where the DOQ would remain close to the surface even if it desorbs and thus could easily adsorb again. The CNT yarn (with mean roughness depth of $1910 \pm 190 \mathrm{~nm}$ )[36] does not trap the dopamine long enough to impair the current-vs-time curve. However, the deep corrugations at PEI/CNT fiber surface $(0.5-1 \mu \mathrm{m}[15]$, Fig. 1E) influence the temporal response remarkably, trapping dopamine and slowing the diffusion to and from the surface, as indicated by the slow rise and fall of the curve (Fig. 2E). Therefore, CNT yarn microelectrodes provide high sensitivity at rapid scan repetition frequency while maintaining a fast temporal response.

This study provides an understanding of which surface properties are important for electrochemical response to neurotransmitters with FSCV. While this study examines the correlation between surface structure and electrochemical performance, the goal for the future would be to develop an integrated model that used surface properties such as surface roughness and surface oxygen content to predict electrochemical performance such as sensitivity and electrocatalytic effects. Even without a full model, understanding the surface property-electrochemical function relationship will facilitate a better evaluation of which potential new nanomaterials to test; for example if the roughness is too large a material would be expected to have a slow response and might not be chosen for electrochemical experiments. Future studies might also examine how nanomaterial structure and electrochemical performance are affected by tissue exposure and other biofouling expected to occur in vivo. 


\section{Conclusions}

Surface properties of CNT fibers produced by different protocols have dramatic effects on the electrochemical performance including sensitivity, selectivity, and response to FSCV frequency. CNT yarn microelectrodes have the highest dopamine and serotonin sensitivity because of the well-aligned, high purity CNTs, and abundant oxygen containing functional groups at the surface. The CA/CNT fiber, which was applied as electrode material for biomolecule detection for the first time, provides high selectivity to dopamine over UA and AA because of the negatively charged surface. CNT yarn and PEI/CNT fiber microelectrodes have an electrochemical response to dopamine that is scan frequency independent due to the surface roughness that allows dopamine to get trapped in the crevices, leading to thin-layer cell conditions. However, the larger surface roughness on the PEI/CNT fiber surface is detrimental to fast temporal response. The studies result in a better understanding of how surface properties influence the electrochemical properties of neurotransmitter detection with FSCV. Thus, surface properties can be tuned in the future and used to predict which carbon nanomaterial-based microelectrodes will be best for detection of different neurotransmitters. 


\section{Acknowledgments}

This research was supported by NIH grant R21 DA037584. Physical surface characterization on the CNT fiber and yarn microelectrodes were conducted at the Center for Nanophase Materials Sciences, which is a DOE Office of Science User Facility (User grant CNMS2016-022). Travel aid to Oak Ridge National Laboratory was supported by ORNL-UVA Travel Award (University of Virginia). 


\section{$\underline{\text { References }}$}

[1] C. Yang, M.E. Denno, P. Pyakurel, B.J. Venton, Recent trends in carbon nanomaterialbased electrochemical sensors for biomolecules: A review, Anal. Chim. Acta. 887 (2015) 17-37. doi:10.1016/j.aca.2015.05.049.

[2] B.E.K. Swamy, B.J. Venton, Carbon nanotube-modified microelectrodes for simultaneous detection of dopamine and serotonin in vivo., Analyst. 132 (2007) 876-84. doi:10.1039/b705552h.

[3] P. Takmakov, M.K. Zachek, R.B. Keithley, P.L. Walsh, C. Donley, G.S. McCarty, et al., Carbon microelectrodes with a renewable surface., Anal. Chem. 82 (2010) 2020-8. doi:10.1021/ac902753x.

[4] N. Xiao, B.J. Venton, Rapid, Sensitive Detection of Neurotransmitters at Microelectrodes Modified with Self-assembled SWCNT Forests, Anal. Chem. 84 (2012) 7816-7822. doi:10.1021/ac301445w.

[5] A.G. Zestos, C.B. Jacobs, E. Trikantzopoulos, A.E. Ross, B.J. Venton, Polyethylenimine Carbon Nanotube Fiber Electrodes for Enhanced Detection of Neurotransmitters, Anal. Chem. 86 (2014) 8568-8575. doi:10.1021/ac5003273.

[6] A.G. Zestos, C. Yang, C.B. Jacobs, D. Hensley, B.J. Venton, Carbon nanospikes grown on metal wires as microelectrode sensors for dopamine, Analyst. 140 (2015) 7283-7292. doi:10.1039/C5AN01467K.

[7] C.B. Jacobs, T.L. Vickrey, B.J. Venton, Functional groups modulate the sensitivity and electron transfer kinetics of neurochemicals at carbon nanotube modified microelectrodes, Analyst. 136 (2011) 3557. doi:10.1039/c0an00854k.

[8] L. Xiang, P. Yu, J. Hao, M. Zhang, L. Zhu, L. Dai, et al., Vertically aligned carbon nanotube-sheathed carbon fibers as pristine microelectrodes for selective monitoring of ascorbate in vivo., Anal. Chem. 86 (2014) 3909-14. doi:10.1021/ac404232h.

[9] C. Yang, C.B. Jacobs, M.D. Nguyen, M. Ganesana, A.G. Zestos, I.N. Ivanov, et al., Carbon Nanotubes Grown on Metal Microelectrodes for the Detection of Dopamine, Anal. Chem. 88 (2016) 645-652. doi:10.1021/acs.analchem.5b01257.

[10] Z. Spitalsky, D. Tasis, K. Papagelis, C. Galiotis, Carbon nanotube-polymer composites: Chemistry, processing, mechanical and electrical properties, Prog. Polym. Sci. 35 (2010) 357-401. doi:10.1016/j.progpolymsci.2009.09.003.

[11] W. Lu, M. Zu, J.H. Byun, B.S. Kim, T.W. Chou, State of the art of carbon nanotube fibers: Opportunities and challenges, Adv. Mater. 24 (2012) 1805-1833. doi:10.1002/adma.201104672.

[12] Z. Li, Z. Liu, H. Sun, C. Gao, Superstructured Assembly of Nanocarbons: Fullerenes, Nanotubes, and Graphene, Chem. Rev. 115 (2015) 7046-7117. doi:10.1021/acs.chemrev.5b00102.

[13] N. Behabtu, C.C. Young, D.E. Tsentalovich, O. Kleinerman, X. Wang, A.W.K. Ma, et al., Strong, Light, Multifunctional Fibers of Carbon Nanotubes with Ultrahigh Conductivity, Science (80-. ). 339 (2013) 182-186. doi:10.1126/science.1228061.

[14] B. Vigolo, A. Pénicaud, C. Coulon, C. Sauder, R. Pailler, C. Journet, et al., Macroscopic Fibers and Ribbons of Oriented Carbon Nanotubes, Science (80-. ). 290 (2000) 1331- 
1334. doi:10.1126/science.290.5495.1331.

[15] E. Muñoz, D.S. Suh, S. Collins, M. Selvidge, A.B. Dalton, B.G. Kim, et al., Highly conducting carbon nanotube/polyethyleneimine composite fibers, Adv. Mater. 17 (2005) 1064-1067. doi:10.1002/adma.200401648.

[16] L.M. Ericson, H. Fan, H. Peng, V.A. Davis, W. Zhou, J. Sulpizio, et al., Macroscopic, Neat, Single-Walled Carbon Nanotube Fibers, Science (80-. ). 305 (2004) 1447-1450. doi:10.1126/science.1101398.

[17] V. a Davis, a N.G. Parra-Vasquez, M.J. Green, P.K. Rai, N. Behabtu, V. Prieto, et al., True solutions of single-walled carbon nanotubes for assembly into macroscopic materials., Nat. Nanotechnol. 4 (2009) 830-834. doi:10.1038/nnano.2009.302.

[18] T. Fujigaya, N. Nakashima, Methodology for Homogeneous Dispersion of Single-walled Carbon Nanotubes by Physical Modification, Polym. J. 40 (2008) 577-589.

doi:10.1295/polymj.PJ2008039.

[19] K. Koziol, J. Vilatela, A. Moisala, M. Motta, P. Cunniff, M. Sennett, et al., HighPerformance Carbon Nanotube Fiber, Science (80-. ). 318 (2007) 1892-1895. doi:10.1126/science.1147635.

[20] M. Zhang, K.R. Atkinson, R.H. Baughman, Multifunctional carbon nanotube yarns by downsizing an ancient technology., Science. 306 (2004) 1358-1361. doi:10.1126/science.1104276.

[21] E. Mayhew, V. Prakash, Thermal conductivity of high performance carbon nanotube yarnlike fibers, J. Appl. Phys. 115 (2014) 174306. doi:10.1063/1.4874737.

[22] K. Jiang, Q. Li, S. Fan, Nanotechnology: spinning continuous carbon nanotube yarns., Nature. 419 (2002) 801. doi:10.1038/419801a.

[23] C.B. Jacobs, I.N. Ivanov, M.D. Nguyen, A.G. Zestos, B.J. Venton, High Temporal Resolution Measurements of Dopamine with Carbon Nanotube Yarn Microelectrodes, Anal. Chem. 86 (2014) 5721-5727. doi:10.1021/ac404050t.

[24] A.C. Schmidt, X. Wang, Y. Zhu, L. a Sombers, Carbon Nanotube Yarn Electrodes for Enhanced Detection of Neurotransmitter Dynamics in Live Brain Tissue., ACS Nano. (2013) 7864-7873. doi:10.1021/nn402857u.

[25] a N.G. Parra-Vasquez, N. Behabtu, M.J. Green, C.L. Pint, C.C. Young, J. Schmidt, et al., Spontaneous dissolution of ultralong single- and multiwalled carbon nanotubes., ACS Nano. 4 (2010) 3969-3978. doi:10.1021/nn100864v.

[26] M. Miao, Electrical conductivity of pure carbon nanotube yarns, Carbon N. Y. 49 (2011) 3755-3761. doi:10.1016/j.carbon.2011.05.008.

[27] S. Amaro, X. Urra, M. Gómez-Choco, V. Obach, Á. Cervera, M. Vargas, et al., Uric acid levels are relevant in patients with stroke treated with thrombolysis, Stroke. 42 (2011) 2833. doi:10.1161/STROKEAHA.110.596528.

[28] M.. Rice, I. Russo-Menna, Differential compartmentalization of brain ascorbate and glutathione between neurons and glia, Neuroscience. 82 (1997) 1213-1223. doi:10.1016/S0306-4522(97)00347-3.

[29] V. Dutt, H. Mottola, Determination of uric acid at the microgram level by a kinetic 
procedure based on a pseudo-induction period, Anal. Chem. (1974) 1777-1781. http://pubs.acs.org/doi/abs/10.1021/ac60348a041 (accessed August 24, 2014).

[30] D.S. Goldstein, C. Holmes, Neuronal source of plasma dopamine., Clin. Chem. 54 (2008) 1864-71. doi:10.1373/clinchem.2008.107193.

[31] C.M. Tallaksen, T. Bøhmer, H. Bell, Concentrations of the water-soluble vitamins thiamin, ascorbic acid, and folic acid in serum and cerebrospinal fluid of healthy individuals., Am. J. Clin. Nutr. 56 (1992) 559-64. http://www.ncbi.nlm.nih.gov/pubmed/1503069.

[32] J.E. Baur, E.W. Kristensen, L.J. May, D.J. Wiedemann, R.M. Wightman, Fast-scan voltammetry of biogenic amines., Anal. Chem. 60 (1988) 1268-72. http://www.pubmedcentral.nih.gov/articlerender.fcgi?artid=3058613\&tool=pmcentrez\&ren dertype $=$ abstract.

[33] B. Jackson, S. Dietz, R. Wightman, Fast-scan cyclic voltammetry of 5-hydroxytryptamine, Anal. Chem. (1995) 1115-1120. http://pubs.acs.org/doi/abs/10.1021/ac00102a015 (accessed November 3, 2014).

[34] B.D. Bath, D.J. Michael, B.J. Trafton, J.D. Joseph, P.L. Runnels, R.M. Wightman, Subsecond adsorption and desorption of dopamine at carbon-fiber microelectrodes., Anal. Chem. 72 (2000) 5994-6002. http://www.ncbi.nlm.nih.gov/pubmed/11140768.

[35] C.W. Atcherley, N.D. Laude, K.L. Parent, M.L. Heien, Fast-scan controlled-adsorption voltammetry for the quantification of absolute concentrations and adsorption dynamics, Langmuir. 29 (2013) 14885-14892. doi:10.1021/la402686s.

[36] C. Yang, E. Trikantzopoulos, M.D. Nguyen, C.B. Jacobs, Y. Wang, M. Mahjouri-Samani, et al., Laser Treated Carbon Nanotube Yarn Microelectrodes for Rapid and Sensitive Detection of Dopamine in Vivo, ACS Sensors. 1 (2016) 508-515. doi:10.1021/acssensors.6b00021.

[37] J.G. Roberts, B.P. Moody, G.S. McCarty, L. a. Sombers, Specific Oxygen-Containing Functional Groups on the Carbon Surface Underlie an Enhanced Sensitivity to Dopamine at Electrochemically Pretreated Carbon Fiber Microelectrodes, Langmuir. 26 (2010) 9116-9122. doi:10.1021/la9048924. 


\section{TOC}
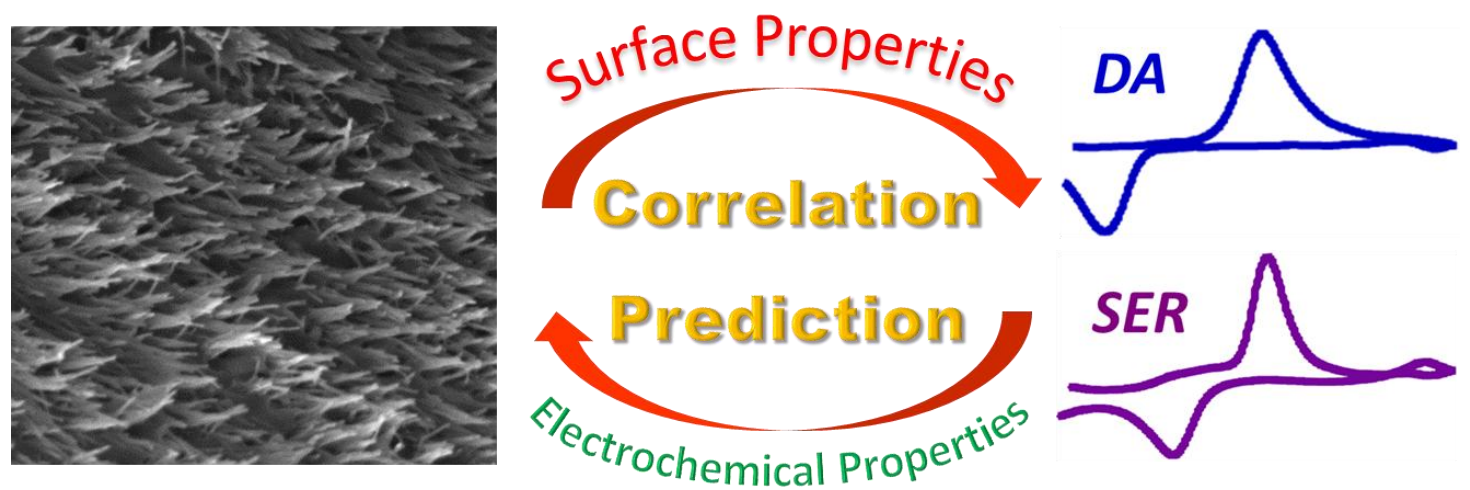